\title{
Téoros
}

Revue de recherche en tourisme

\section{L'industrie de croisière dans la Caraïbe} Facteur de développement ou pâle reflet de la mondialisation?

\section{Kinvi Logossah}

Volume 26, numéro 1, printemps 2007

Tourisme dans la Caraïbe : logiques régionales et perspectives de développement

URI : https://id.erudit.org/iderudit/1070993ar

DOI : https://doi.org/10.7202/1070993ar

Aller au sommaire du numéro

\section{Éditeur(s)}

Université du Québec à Montréal

ISSN

0712-8657 (imprimé)

1923-2705 (numérique)

Découvrir la revue

Citer cet article

Logossah, K. (2007). L'industrie de croisière dans la Caraïbe : facteur de développement ou pâle reflet de la mondialisation ? Téoros, 26(1), 33-39. https://doi.org/10.7202/1070993ar 


\section{L'industrie de croisière dans la Caraïbe Facteur de développement ou pâle reflet de la mondialisation?}

\section{Kinvi Logossah}

Alors que les caractéristiques naturelles des petites économies insulaires, notamment l'isolement, l'éloignement, l'exiguiité spatiale et démographique, l'exposition aux risques naturels sismiques, cycloniques, semblent les condamner, à la lumière des théories économiques, à demeurer sur une trajectoire de croissance basse et donc dans le sous-développement, en raison soit de la faiblesse de la demande interne ou des économies d'échelle (Grossman et Helpman, 1991 ; Aghion et Howitt, 1998), soit des coûts de transport ou de communication élevés, soit de leur pouvoir d'attraction réduit des investissements directs étrangers (Logossah et Maupertuis, 2007), les faits ne semblent pas confirmer de telles analyses.

En effet, étudiant, sur la période 1960-1995, 157 pays qu'ils ont scindés en cinq groupes, à savoir : pays de l'OCDE (Organisation de coopération et de développement économiques), pays pétroliers, petits pays, petits pays à spécialisation touristique et pays en développement, Brau et al. (2003: 8-18) montrent que les petits pays à spécialisation touristique ont connu le taux de croissance le plus élevé ${ }^{1}$ dans le monde. En outre, recourant à plusieurs séries de tests économétriques, sur la période 1980-1995, ces auteurs attestent de l'impact positif significatif de la spécialisation touristique sur la croissance dans les petits pays insulaires. De même, Marques (2005: 215), testant économétriquement l'effet des recettes touristiques sur le PIB (produit intérieur brut) pour 27 petites économies insulaires de la Caraïbe sur la période 1980-2000, obtient un coefficient de corrélation très fort entre les deux variables.
Si la spécialisation touristique se présente ainsi comme un déterminant majeur de la croissance dans les petites économies insulaires, notamment de la Caraïbe, c'est principalement parce qu'elle transforme l'essentiel des handicaps naturels de ces économies (isolement, éloignement, insularité) en ressources productives touristiques, au point de leur conférer un avantage important.

Toutefois, les analyses précédentes ont été menées en référence au secteur touristique pris globalement ou en référence au tourisme de séjour dont les canaux d'impact sur les économies locales sont nombreux et significatifs. Peu d'études ont par ailleurs distingué le rôle du type de spécialisation touristique dans la croissance et le développement des petites économies insulaires, notamment le rôle du tourisme de croisière sur le développement.

Or, le tourisme de croisière connaît un boom sans précédent dans cette région qui concentre de surcroît près de la moitié des flux mondiaux de croisiéristes et les autorités régionales l'entrevoient comme un facteur de développement durable potentiel dans la Caraïbe. Néanmoins, la nature particulière de cette industrie amène à s'interroger sur la pertinence d'une telle stratégie: d'une part les acteurs de l'offre, les compagnies de croisière, sont complètement "hors " de la région ; d'autre part, autant les canaux d'intégration de l'activité de croisière à l'économie de la région que les ressources financières injectées dans l'économie caribéenne sont ténus alors que d'importantes externalités négatives dues à l'industrie pourraient se retrouver à la charge de la région. D'où la question des enjeux de l'activité de croisière dans la Caraïbe : outil de développement fondé sur une stratégie des agents économiques caribéens ou pâle reflet de la mondialisation galopante?
Cette interrogation est au cœur de la réflexion menée dans le présent article, qui tente de cerner les enjeux économiques du tourisme de croisière dans la Caraïbe. Pour cela sont analysés la contribution de l'activité de croisière à l'économie caribéenne, le rôle et les stratégies des acteurs, ainsi que la durabilité de cette activité.

\section{L'industrie de croisière dans la Caraïbe : quelle contribution à l'économie régionale?}

\section{La croisière: une activité centrée sur la Caraïbe}

Le tourisme est l'une des industries à plus forte croissance dans le monde: en effet, sur les trois dernières décennies, la demande touristique mondiale est passée de 165,8 millions d'arrivées en 1970 à 763,2 millions en 2004 (CTO, 2006b: 7), progressant au rythme annuel moyen de 3,7\% sur la période. Bien que la région Carailbe, dans son ensemble, ne reçoive qu'une part faible de cette demande mondiale (2,5\% en 1970, 2,9\% en 2004 et 2,3\% en 2005) (WTO, 2006: 7 ; Lester et Weeden, 2004 : 39-41), son marché connaît une croissance plus rapide: le taux de croissance annuel moyen des flux de touristes dans la Caraïbe s'établit à 4,2 \% sur la période 1970-2004.

En matière de croisière, au contraire, l'essentiel de la demande mondiale reste concentré dans la Caraïbe qui détient $46,8 \%$ de la capacité d'offre en lit/ jour de l'industrie en 2004, contre 12,5\% pour la Méditerranée et 9,8\% pour l'Europe (CTO, 2006b : 61). En outre, la croisière dans la Caraïbe constitue, selon une enquête de la Cruise Line Industrie Association (CLIA), le rêve de 40 \% des vacanciers nord-américains (principal foyer d'émission de croisiéristes dans le monde), loin devant la Floride (17\%) et Hawaï (11\%) (Dowling, 2006 : 6). 
Dans l'ensemble de la Caraïbe, le marché de croisière tend progressivement à égaler en termes de masse de visiteurs celui du tourisme de séjour. Ainsi, alors qu'en 1980 les croisiéristes étaient de 3,8 millions d'individus contre 6,9 millions de touristes de séjour, ces chiffres sont passés respectivement de 7,7 millions contre 12,8 millions en 1990, puis de 14,5 millions contre 20,3 millions en 2000, pour s'établir à 19,8 millions contre 21,8 millions en 2004 (CTO, 2006a). Ainsi, par rapport aux touristes de séjour, les croisiéristes représentent dans la Caraïbe $55 \%$ en $1980,60,5 \%$ en 1990 , $71,6 \%$ en 2000 et $91 \%$ en 2004 .

Tel qu'on peut le constater à la lecture du tableau 1, d'après nos calculs, dans 13 des 34 pays de la Caraïbe, le flux des touristes de croisière excède celui des touristes de séjour en 2004. C'est le cas à Cozumel qui a reçu 7 fois plus de croisiéristes que de touristes de séjour ; c'est aussi le cas aux îles Caïman avec 6 fois plus de croisiéristes que de touristes de séjour ou à la Dominique avec 4 fois plus, au Belize 2 fois plus, aux Bahamas, à Antigua et Barbuda, à Saint-Martin, à Saint-Kitts et Nevis, 2 fois plus.

Cette étendue du marché de la croisière s'accompagne aussi d'une progression plus rapide du flux des arrivées comparé à celle des touristes de séjour. En effet, le marché des touristes de séjour a cru au taux annuel moyen de 3,2 \% sur la période 1980-2004, alors que, sur la même période, le flux des croisiéristes a augmenté au taux annuel moyen de 6,2\%, soit du double.

Bien que le marché de croisière s'étende de plus en plus au monde entier, l'essentiel des touristes de croisière dans la Caraïbe provient d'Amérique du Nord, les États-unis constituant jusqu'à environ $80 \%$ de la demande. Par ailleurs, comme cela apparaît au graphique 1 , le marché de croisière caribéen reste fortement concentré dans l'espace : sept destinations reçoivent près des trois quarts des croisiéristes (CTO, 2006b: 20) : il s'agit des Bahamas, de Cozumel, des îles Vierges américaines, des îles Caïmans, de Saint-Martin, de Puerto Rico et de la Jamaïque; alors que quatre de ces destinations, notamment les Bahamas, Cozumel, les îles Vierges américaines et les îles Caïmans reçoivent plus de la moitié des croisiéristes de la Caraïbe (52,8 \%).
Sur le plan des recettes touristes et lorsqu'on considère l'industrie du tourisme de séjour, la Caraïbe reçoit globalement 3,5 milliards USD en 1980. Ces recettes augmentent à 21,6 milliards USD en 2004, ce qui correspond, sur la période 1980-2004, à une progression au taux annuel moyen de $5 \%$. Ce taux est supérieur à celui observé globalement dans le monde sur la même période, où les recettes du tourisme de séjour ont cru au rythme annuel moyen de 4,8\%. Cette croissance plus rapide des recettes touristiques de la région traduit une certaine " efficacité » puisque, avec 2,9\% de la part mondiale des touristes de séjour en 2004, la Caraïbe obtient 3,5\% des dépenses mondiales de ces touristes.

\section{Tableau 1}

Flux de touristes dans la Caraïbe en 2004

\begin{tabular}{l|c|c|c}
\hline \multirow{2}{*}{ Pays } & \multicolumn{2}{|c|}{ Flux des touristes (milliers) } & Part Croisiéristes/touristes de séjour \\
\cline { 2 - 3 } & Touristes de séjour & Croisiéristes & \\
\hline Bahamas & 1561,3 & 3360 & 2,1 \\
Antigua et Barbuda & 245,8 & 522,8 & 2,1 \\
Îles Vierges britanniques & 304,5 & 466,6 & 1,5 \\
Dominique & 80,1 & 383,6 & 4,7 \\
Saint-Kitts et Nevis & 91,8 & 260,2 & 2,8 \\
Saint-Martin & 475 & 1348,5 & 2,8 \\
Îles Vierges américaines & 543,6 & 1964,7 & 3,6 \\
Barbade & 552 & 721,3 & 1,30 \\
Grenade & 133,9 & 229,8 & 1,7 \\
Sainte-Lucie & 298,4 & 481,3 & 1,6 \\
Belize & 230,8 & 851,4 & 3,6 \\
Îles Caïmans & 259,9 & 1693,3 & 6,5 \\
Cozumel (Mexique) & 368,6 & 2862 & 7,7 \\
\hline
\end{tabular}

Source : Caribbean Tourism Association (CTO), $2006 \mathrm{~b}$.

\section{Graphique 1}

Principaux marchés de croisière dans la Caraïbe en 2005. Part des flux de croisiéristes en \%

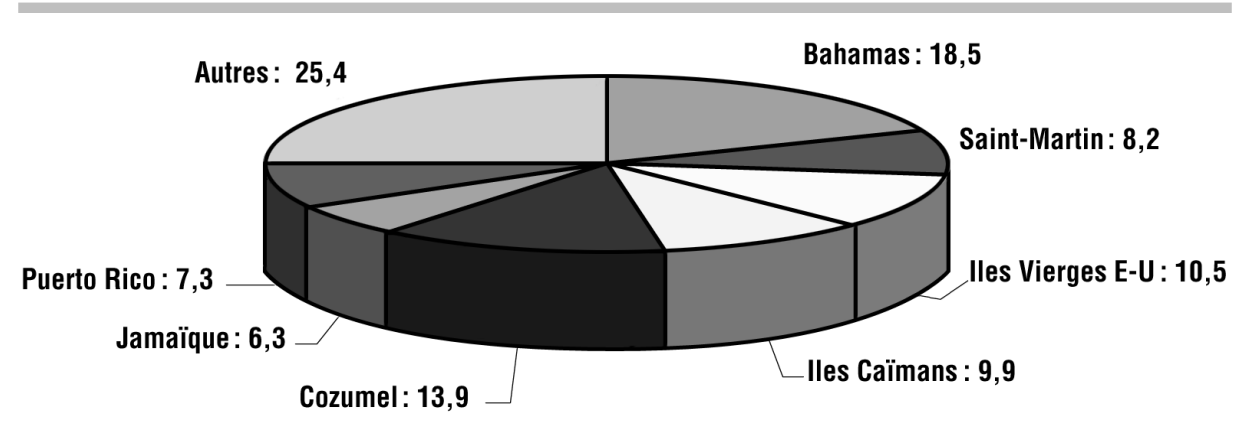

Source : Nos calculs à partir des données de la Caribbean Tourism Organization / CTO (2006) - tableau 2.
Au regard des types de spécialisation touristique, la Caraïbe reste plus « efficace» en matière de tourisme de séjour: ainsi, en 2004, alors que les touristes de croisière y représentent $91 \%$ des touristes de séjour, leurs dépenses ne représentent que $14,1 \%$ des dépenses des touristes de séjour, soit environ 3 milliards USD contre 21,6 milliards USD.

\section{Le tourisme de croisière dans la Caraïbe : portée et limites}

Cette relative « inefficacité » de l'industrie de croisière s'expliquerait par le nombre restreint de ses canaux d'impact sur l'économie caribéenne. En effet, les recettes 
dont bénéficient directement les économies de la région de l'industrie proviennent essentiellement :

- des dépenses des croisiéristes visitant les pays ;

- des approvisionnements de l'équipage dans les pays ;

- de la taxe passager que versent les compagnies ;

- des droits de stationnement des bateaux au port.

Or, à partir des années 1990, les nouvelles stratégies d'exploitation des lignes de croisière tendent de plus en plus à maximiser le nombre de touristes qui restent à bord des paquebots lors des escales dans les différents pays et à minimiser le temps que les croisiéristes passent à visiter ces pays. En conséquence, leurs dépenses terrestres n'augmentent que très peu et tendent à stagner ou même à diminuer. Ainsi, la dépense moyenne mondiale par croisiériste visitant les territoires se monte à 36 \$ en 1987, à 34 \$ en 1990, à 23,1 \$ en 1996 et à 16,4 \$ en 2002 (CMT, 2003b : 59). Pour l'ensemble de la Caraïbe, cette dépense moyenne s'évalue à $34 \$$ en 1993 et à 25,1 \$ en 2002 (CMT, 2003b : 59) ; à la Martinique, de 28,50 euros en 1992, elle passe à 21,10 euros en 2000, puis à 13,80 euros en 2003 (CMT, 2003a: 192).

\section{Les Départements français d'Amérique (DFA): des économies peu touristiques}

Dans la Carailbe, les DFA regroupent essentiellement deux îles : la Guadeloupe et ses dépendances (environ 1700 kilomètres carrés pour 438800 habitants) et la Martinique (1100 kilomètres carrés pour 391000 habitants). Par opposition aux autres îles de la Caraïbe, l'industrie touristique joue un rôle marginal dans les économies des DFA, encore largement administrées : les transferts publics, français et européens, restent l'un des déterminants majeurs de la richesse dans ces économies. Les données disponibles montrent ainsi qu'à la Martinique, sur la période 1987-2001, le secteur touristique dans sa globalité ne contribue en moyenne qu'à hauteur de 4,4\% du PIB, bien que sur la période 2000-2003, par exemple, les recettes touristiques s'élèvent en moyenne à $72 \%$ des exportations (CMT, 2003b : 105-107 ; DRCE, 2004) ; par ailleurs, à la Martinique, le secteur touristique ne contribue qu'à $14 \%$ des emplois des services et à $5 \%$ de l'emploi global de l'île.
Ensemble, les DFA représentent 6,1\% des touristes de séjour de la Caraïbe en 2004, soit un flux de 1,334 millions de touristes (CTO, 2006b) ; cette part se répartit à raison de $4 \%$ pour la Guadeloupe (863 300 touristes) et de 2,1\% pour la Martinique (470 900 touristes) pour l'année. Sur la période récente, 2000-2004, les flux ont progressé moins vite dans les DFA $(0,02 \%)$ que dans l'ensemble de la Caraïbe $(1,8 \%)$; cette situation s'explique par le recul des flux de visiteurs de séjour à la Martinique $(-2,6 \%$ en moyenne l'an sur la période), alors que ceux de la Guadeloupe augmentaient au rythme annuel moyen de 1,7 \% (idem).

Le tourisme de croisière est encore plus ténu dans les DFA: en 2003, par exemple, ceux-ci n'ont reçu ensemble que 463600 croisiéristes, soit 2,6\% des flux de la Caraïbe, cette part se répartissant à raison de 1,5\% pour la Martinique et de 1,1\% pour la Guadeloupe. En outre, sur la période 2000-2003, les DFA ont connu un reflux des croisiéristes au rythme annuel moyen de 10,5\%, ce taux de reflux s'établissant à 1,7 \% l'an en moyenne pour la Guadeloupe contre 31,2\% pour la Martinique, alors que dans l'ensemble de la Caraỉbe, sur la même période, la progression était de 7,3\% l'an en moyenne. Enfin, dans les DFA, le tourisme de séjour reste dominant: en 2003, dans l'ensemble des DFA, les flux de touristes de séjour font 2,7 fois ceux des croisiéristes, cette proportion étant de 4 fois en Guadeloupe, de 1,7 fois en Martinique et de 1,1 fois dans la Caraïbe.

S'agissant des recettes touristiques, tous visiteurs confondus, les DFA ont engrangé en 2004 663,1 millions USD, dont $56 \%$ pour la Guadeloupe et $44 \%$ pour la Martinique, soit 3,1\% des recettes touristiques de la Caraïbe (CTO, 2006b). Les données disponibles (CMT, 2003b : 106) montrent que, sur la période 2000-2003, la croisière a rapporté annuellement à la Martinique environ 9,6 millions USD en recettes directes, ce qui représente, sur la période, 3,5\% des recettes directes du tourisme de séjour en moyenne chaque année.

Au total, le tourisme dans les DFA reste encore largement naturel : il n'existe pas de stratégie touristique clairement définie et l'offre touristique repose principalement sur le traditionnel "soleil-mer-plage»; plus des trois quarts des touristes de séjour sont originaires de la France et plus des deux tiers des croisiéristes sont nord-américains.
Pour l'ensemble de la Caraïbe, on estime qu'une part significative des dépenses des croisiéristes porte sur des biens importés offerts principalement dans les boutiques hors taxe. Cette part, dans l'ensemble de la Caraïbe, selon Pattulo (cité dans Seidl et al., 2006 : 218), s'élèverait à $45 \%-67 \%$, tandis que $17 \%$ des dépenses sont consacrées aux attractions et excursions et $8 \%$ aux repas. Au Costa Rica, en 2003, les études montrent que de $64 \%$ à $72 \%$ des dépenses des croisiéristes portent sur des biens importés (Seidl et al., 2006 : 218). En Martinique, en 2003, les enquêtes du CMT montrent que $57 \%$ des dépenses des croisiéristes portent sur le shopping, dont $17 \%$ vont aux parfums et $11,1 \%$ aux vêtements.

Il importe de souligner cependant que l'impact économique de l'industrie de croisière dans la Caraïbe en termes de revenus ne se limite pas aux seules ressources financières directement injectées dans les économies ; il englobe en outre les revenus additionnels dus aux effets d'entraînement sur les diverses branches économiques locales et aux effets induits liés aux dépenses des agents. Un essai d'évaluation réalisé à la demande de la Florida Caribbean Cruise Association (Pricewaterhousecoopers et Brea, 2001) indique que pour l'ensemble de la Caraïbe, en 2000, les recettes directes générées par la croisière s'élèvent à 1,4 milliard USD et elles ont elles-mêmes engendré 1,2 milliard USD, puis 34000 emplois directs et 26000 emplois indirects; les mêmes calculs effectués pour les années 1992 et 1995 donnent respectivement 1,8 milliard USD et 2,3 milliards USD de revenus directs et indirects générés par la croisière dans la Caraïbe; ce qui conduit à créer au total 35500 emplois en 1992 et 48000 en 1995. Toutefois, cette étude repose sur l'hypothèse d'un multiplicateur de dépenses des croisiéristes de 2,5, que les chercheurs estiment invraisemblable (Pattulo, cité dans Seidl et al., 2006). Par exemple, les multiplicateurs de dépenses des croisiéristes ont été évalués à 1,43 pour les effets sur l'emploi, à 1,88 pour les effets sur la valeur ajoutée et à 1,62 pour les effets sur les salaires pour la Floride (Braun et al. , 2002) ; pour le Costa Rica, le multiplicateur de dépenses des croisiéristes est évalué à 1,4 (Seidl et al., 2006).

Les impacts économiques, directs et indirects, de l'industrie de croisière étant ténus, les autorités touristiques caribéennes 
(Caribbean Tourism Organization / CTO) misent sur la capacité des territoires à transformer les croisiéristes qui visitent la région lors des diverses escales portuaires en touristes de séjour futurs. II est à noter que l'industrie pourrait, en effet, se révéler d'un impact économique notable si une part significative des anciens croisiéristes devenaient des touristes de séjour effectifs, vu l'importance de ce type de touriste pour l'économie régionale. Or, des études commandées par la Florida Caribbean Cruise Association (FCCA) ont montré qu'environ la moitié des croisiéristes qui visitent les îles caribéennes envisagent y retourner pour des séjours (Wood, 2004: 167). À la Martinique, les enquêtes récurrentes de l'Agence régionale de développement touristique de la Martinique (devenue le Comité martiniquais du tourisme en 2003) établissent que $69,2 \%$ des croisiéristes en 1990, 73 \% en 1996 et 70,3 \% en 2002 envisagent revenir à la Martinique en tant que touristes de séjour (CMT, 2003b : 55). Toutefois, si un responsable de la FCCA estimait que $25 \%$ des croisiéristes le font effectivement, son opinion n'est pas fondée sur des études objectives, qui d'ailleurs font cruellement défaut sur ce sujet important (Wood, 2004 : 167). Néanmoins, une étude menée aux Bahamas par Wilkinson (cité par Wood, 2004 : 167) établit que le flux de touristes de séjour de l'année 1996 est constitué à $6,2 \%$ de croisiéristes ayant précédem-

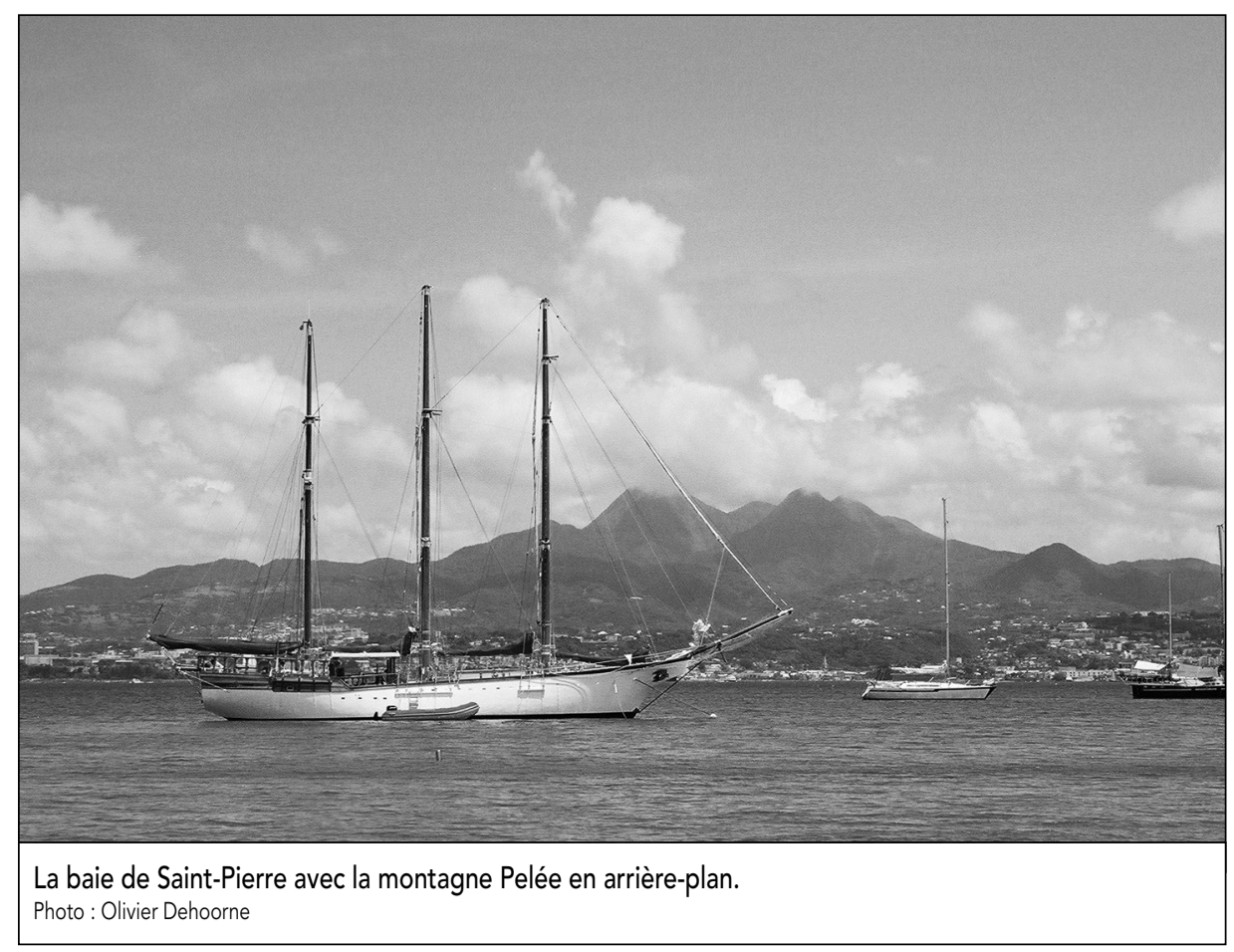

ment visité l'île. À la Martinique, les enquêtes ont établi que $13,1 \%$ des touristes de séjour de l'île en 1990, 13,8 \% en 1995, $20,6 \%$ en 2000 et 22,7\% en 2003 avaient déjà visité l'île (CMT, 2003b : 83) sans que l'on sache s'ils étaient préalablement des touristes de séjour ou des croisiéristes. Or, les mêmes enquêtes révèlent des proportions importantes de touristes de séjour ayant exprimé l'intention de visiter à nouveau l'île, soit 85,6 \% en 1990 et 88,3 \% en 1995 (CMT, 2003b : 101), des chiffres supérieurs à ceux relatifs aux croisiéristes. Par ailleurs, l'étude de Morisson et al. (cités dans Wood, 2004: 167) montre que les croisiéristes sont en général des voyageurs spécifiques peu enclins à se convertir en touristes de séjour, ce que tend à confirmer une enquête récente de la Cruise Line Industrie Association qui révèle que $85 \%$ des croisiéristes projettent de refaire une croisière ultérieurement (Dowling, 2006: 6). Enfin, il importe de souligner que les nouvelles stratégies des compagnies de croisière, faisant de plus en plus des bateaux de croisière des destinations en soi et tendant à inciter davantage au retour des croisiéristes vers «les destinations paquebots », réduiront de plus en plus le taux de conversion des croisiéristes en touristes de séjour dans la Caraïbe, limitant d'autant l'impact économique de la croisière dans la région.

Dans les années 1990 et 2000, la taille des paquebots est en effet devenue un élément important de concurrence entre les lignes de croisière et la tendance est à l'exploitation de grands et méga-paquebots (plus de 50 tonnes et 1200 passagers : Dowling, $2006: 7$ ). Ceux-ci permettent de répondre à la croissance rapide de la demande de croisière et d'exploiter les économies d'échelle. En outre, la grande taille permet d'offrir aux passagers une gamme plus étendue d'activités à bord. Cependant, au-delà de la taille des paquebots, les innovations en matière d'équipement et d'offre de produits et services aux passagers à bord sont devenues les terrains majeurs de rivalité entre compagnies et groupes de croisière. D'importants efforts sont alors déployés afin que les produits, matériels ou immatériels, et les activités répondent aux attentes, aux besoins, aux désirs et aux rêves des passagers. Quels sont ces besoins? Une enquête récente de la Cruise Line Industrie Association (CLIA) auprès des croisiéristes permet d'en dessiner les contours : le croisiériste type n'est plus, 
comme naguère, le retraité, mais un jeune en activité, âgé de 35 à 54 ans, marié, dont le revenu annuel moyen est d'environ 50000 USD et dont une proportion non négligeable (16\%) est souvent accompagnée d'enfants de moins de 18 ans (Dowling, 2006 : 5). Ces données restent conformes à celles des enquêtes récurrentes menées (CMT, 2003b: 41-44). L'enquête de la CLIA révèle en outre que le croisiériste type d'aujourd'hui est un voyageur fréquent (3,8 voyages par an) qui recherche le loisir, le divertissement, le luxe, l'exotisme, l'exclusivité, les produits de santé (Dowling, 2006: 5), une gamme de choix étendue, etc.

Ces caractéristiques des voyageurs expliquent largement celles de la nouvelle génération des bateaux de croisière. Ces bateaux incorporent de plus en plus des équipements modernes complexes: salles de conférences gigantesques ultra équipées avec possibilité d'interprétation en plusieurs langues, cabines avec balcons privés, centre d'étude, planétarium, centre de santé, centre de thalassothérapie, centre d'affaires, boutiques, casinos, discothèques, ascenseurs en verre, salles de spectacle, salles de sports collectifs et individuels (basket, golf, etc.), restaurants variés, restauration diététique, etc. En somme, tous les équipements de tourisme de divertissement et de loisir, d'affaires, de santé, de tourisme culturel, etc. que l'on trouve dans les stations touristiques terrestres. Dans les années récentes, la tendance est à l'offre de séjours courts, d'une semaine, moins onéreux, qui répondent aux besoins de touristes en activité et leur permettent de faire une première expérience de croisière. En outre, on assiste à une différenciation accrue des produits offerts aux clients : les stratégies marketing mettent de plus en plus l'accent sur les marques des lignes qui se spécialisent dans des positionnements exclusifs; alors qu'initialement les compagnies de croisière faisaient la promotion des ports d'appel exotiques comme principal élément du produit, les campagnes marketing mettent désormais davantage l'accent sur les produits disponibles à bord. Les croisières à thème deviennent décisives et de plus en plus la norme et presque toutes les compagnies en proposent aujourd'hui (Dowling, 2006 : 11), à la suite du succès spectaculaire de Carnival Cruises qui avait lancé, dans les années 1980, son fun ship qui affichait l'importance des divertissements à bord. Carnival Cruises a également innové avec sa "garantie vacance » (le passager non satisfait peut des- cendre au prochain port et se faire rembourser) qui est devenue un révélateur majeur de la qualité de son offre de croisière, dont les effets marketing sont importants. Disney Cruises pour sa part "appelle », entre autres, les familles avec enfants.

Les paquebots de croisière sont ainsi devenus des destinations touristiques en soi, reléguant au second plan les territoires auxquels ils se sont substitués au premier plan dans le marketing et le séjour réel des croisiéristes. Les innovations, les différenciations, ajoutées à l'intensification des activités à bord, permettent à ces nouvelles destinations touristiques de maximiser le nombre de passagers qui restent à bord lors des escales portuaires et de maximiser le temps globalement passé par les passagers à bord ainsi que leurs dépenses à bord.

Les paquebots sont ainsi devenus de nouveaux «territoires touristiques, sans terre ", dont une autre des caractéristiques, et non des moindres, est la faculté de pouvoir capter les aménités géographiques propres

\section{Schéma 1}

Les acteurs de l'offre de croisière dans la Caraïbe

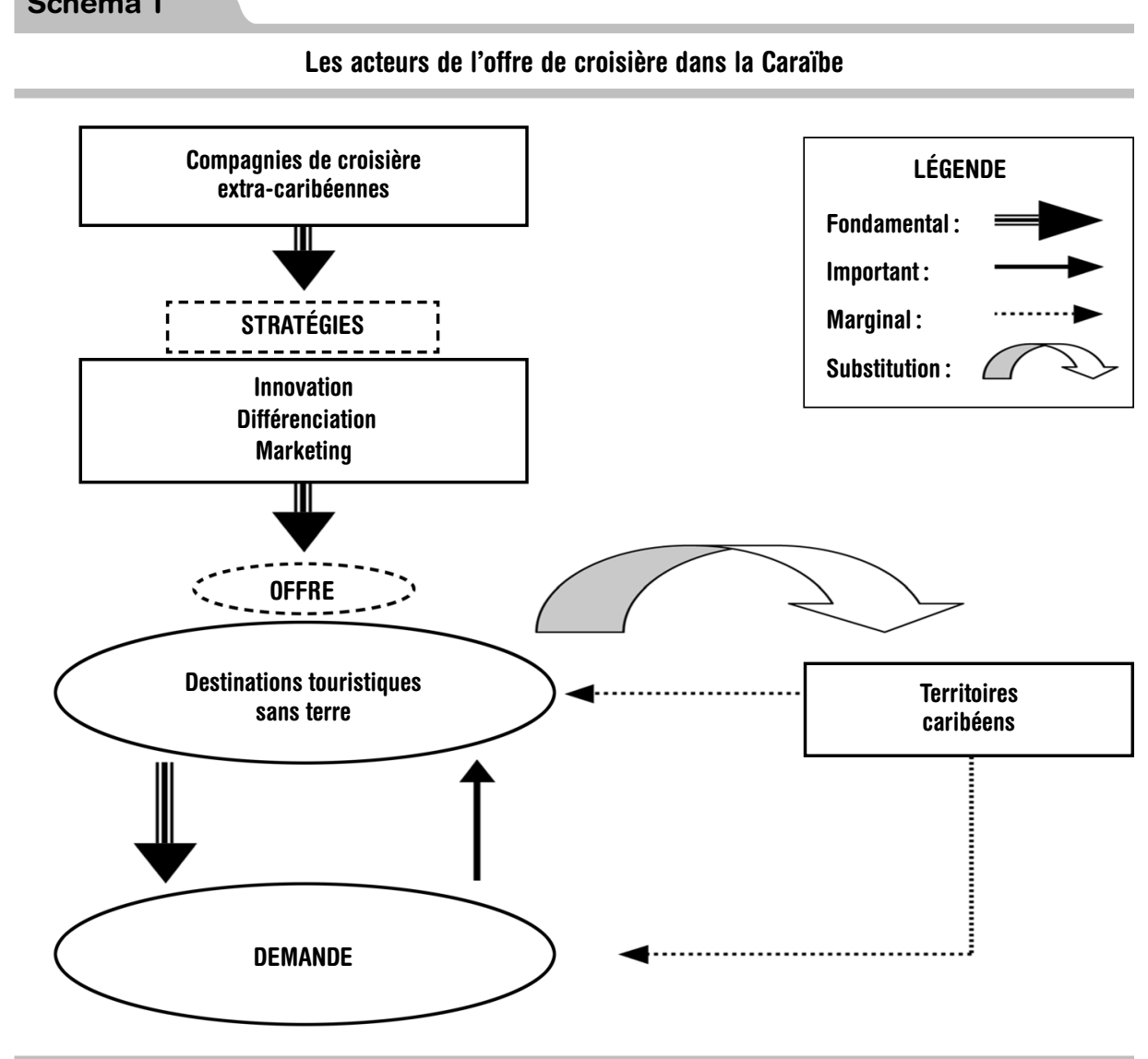

Source : Auteur. aux territoires terrestres, notamment le climat chaud (soleil) qui est l'un des déterminants majeurs de la demande mondiale de croisière : grâce à leur mobilité, les bateaux de croisière se localisent selon la demande en fonction du climat. Ainsi, en hiver (novembre à avril) la majeure partie de la flotte mondiale est déployée dans la Caraïbe alors qu'elle vogue ailleurs en été (mai à octobre), soit en Europe du Nord, en Méditerranée, en Alaska. Les compagnies de croisière échappent en conséquence à la saisonnalité du tourisme dont souffrent en général les destinations terrestres.

Ainsi, les territoires terrestres caribéens ne jouent qu'un rôle marginal dans les stratégies d'offre touristique qui répondent à l'évolution de la demande de croisière, mais l'orientent aussi (l'offre crée également le désir et le besoin chez le client !). Les compagnies ont en définitive un rôle prééminent : elles sont mâ̂tres du marché caribéen de croisière qu'elles orientent en fonction de leurs intérêts, comme on peut le voir au schéma 1. 
Dès lors, l'impact économique ténu de la croisière dans la Caraïbe se révèle in fine comme le corollaire direct du rôle marginal de la région dans la stratégie d'offre de l'industrie. On peut en conséquence s'interroger sur la pertinence d'une stratégie de développement fondée sur le tourisme de croisière.

\section{Un facteur de développement durable?}

L'industrie de croisière dans la Caraïbe s'avère une activité florissante. Par exemple, en 2002, Carnival Coporation dégage un taux de profit de $30 \%$ correspondant à une valeur de 1,02 milliard USD ; Royal Caribbean dégage un taux de profit de $9 \%$ correspondant au montant global de 254 millions USD; ces chiffres se montent à $14 \%$ et à 301 millions USD pour P\&O Princess, à $6 \%$ et 82,6 millions USD pour Star Cruises, soit un total de 1,66 milliard USD de profit pour les quatre compagnies et un taux de profit moyen de $17 \%$ (Klein, 2003, cité dans Seidl et al., 2006 : 216). Cette profitabilité substantielle de l'industrie est la résultante non seulement de ses conditions de base (demande en forte expansion, régime de pavillon de complaisance, etc.), mais également de sa structure (duopole dont les produits sont différenciés, barrières importantes à l'entrée constituées des coûts fixes d'investissements préalables), puis des stratégies des compagnies (innovation, création à bord de produits concurrençant le tourisme terrestre de séjour, marketing offensif, etc.). Par exemple, les stratégies des compagnies ont conduit à des taux d'occupation effectifs des paquebots se situant à 90-95 \% en moyenne sur l'année (ibid.), tandis que l'enregistrement sous le régime de pavillon de complaisance permet de minimiser à outrance les coûts de production : coûts salariaux, impôts et taxes, coûts de traitement et gestion des déchets, etc.

Mais ces profits ne rémunèrent quasi exclusivement que des capitaux extra-caribéens. Par ailleurs, comme nous l'avons indiqué plus haut, l'impact économique de l'industrie de croisière sur l'économie de la Caraïbe s'avère faible, en tout cas nettement inférieur à celui du tourisme de séjour (Seidl et al., 2006 : 222). La région bénéficie ainsi marginalement des retombées positives de l'industrie de croisière, mais pourrait, en revanche, en supporter l'essentiel des coûts externes.
D'abord sur le plan social, nous avons noté que l'industrie est à l'origine de peu d'emplois directs caribéens à bord et dans les activités au sol ; en outre, on ne connaît pas la répartition de ces emplois selon le genre. Par ailleurs, parce que les paquebots battent pavillon de complaisance panaméen et libérien principalement et qu'ils sont de ce fait placés sous la réglementation de ces pays en matière de travail, de fiscalité, etc. il en résulte que le personnel employé à bord est mal rétribué (Wood, 2005: 166) et a de mauvaises conditions de travail: travail 7 jours sur 7,12 heures et plus par jour pendant 4 à 6 mois, sans un seul jour de repos ; syndicalisation interdite à bord; postes de travail attribués hiérarchiquement en fonction de l'origine ethnique des travailleurs (Wood, $2005: 8)$.

Sur le plan environnemental, le régime de pavillon de complaisance qui place les compagnies de croisière sous la réglementation du Panama et du Libéria principalement, leur permet de faire peu cas de l'observation des normes en vigueur (Wood, 2004 : 159-162 ; 2005 : 6-10 ; Thomas, 2004 : 539-541). Or, on considère que l'activité de croisière génère $77 \%$ de la pollution marine mondiale (Wood, 2004 : 162). En effet, les bateaux de croisière ont un grand potentiel de pollution. Ainsi, selon Ocean Conservancy (2002: 3), la quantité de déchets que génère quotidiennement un grand bateau de croisière se monte à: 37000 gallons $^{2}$ d'eaux grasses, 30 000 gallons de vidange, 255000 gallons

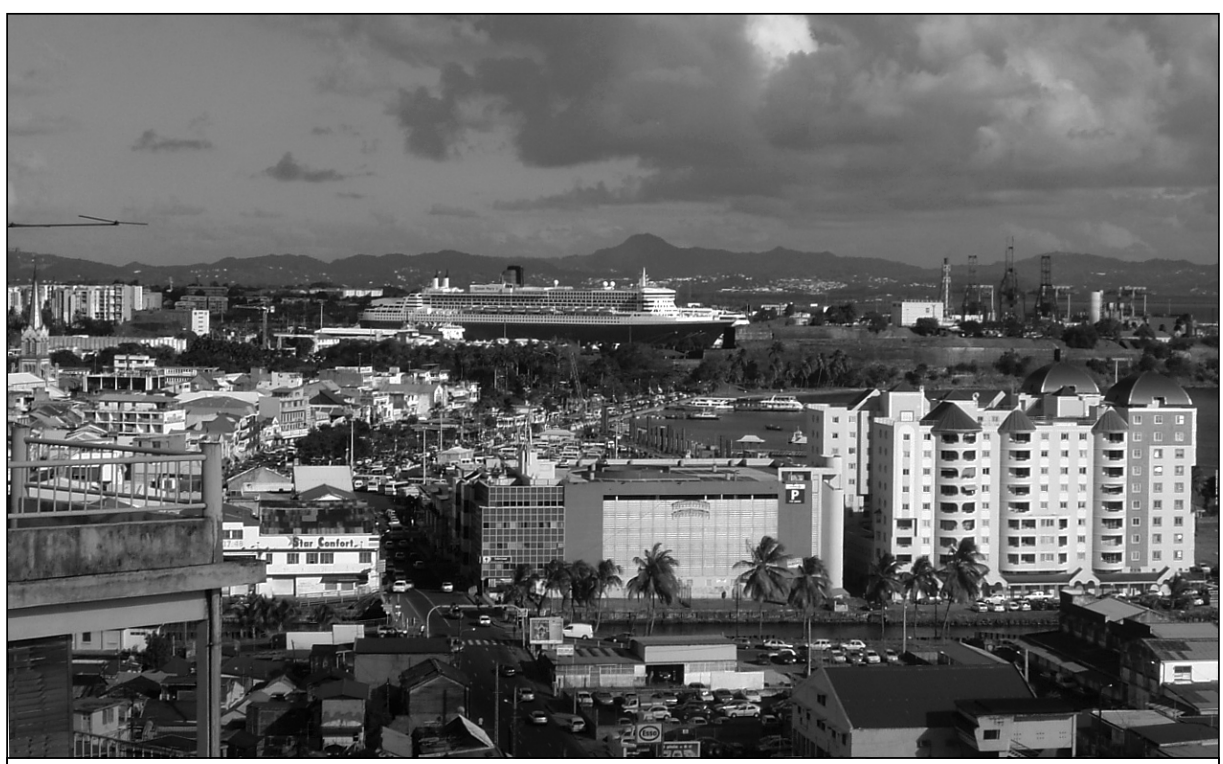

Le Queen Mary dans le port de Fort-de-France en 2004.

Photo : Olivier Dehoorne d'eaux usées, 15 gallons de produits chimiques toxiques, des dizaines de milliers de gallons de lest, 7 tonnes d'ordures et de déchets solides, un niveau de pollution de l'air, généré par des moteurs diesel dans les ports d'escale, équivalent à celui de milliers d'automobiles. Et tout cela, excepté néanmoins les plastiques et les huiles, peut être jeté en toute légalité dans les eaux internationales (Wood, 2004 ; 2005; Lester et Weeden, 2004 : 41-43). Bien que les eaux territoriales, entre 3 miles et 12 miles des rivages parfois, soient placées sous réglementation nationale des pays, on peut estimer que les déchets déversés au large des eaux internationales finiront en partie par être transportés par les courants jusque dans les eaux territoriales et sur les rivages: les territoires risquent en définitive de supporter les coûts de la pollution. Dans la Caraïbe, bien qu'il manque des évaluations précises des dégradations environnementales dues à l'activité de croisière, on peut mentionner quelques cas concrets: afin de recevoir les méga-paquebots de croisière, les territoires investissent dans la construction ou l'extension de ports ; bien que cela puisse générer des revenus et créer des emplois, ces opérations se soldent également par une dégradation de l'environnement et une pollution visuelle; dans les environs de Georgetown et de Grand Cayman par exemple, on a signalé la destruction de 300 acres de récifs coraux par les paquebots de croisière (Wood, 2004 : 164) ; par ailleurs, plusieurs cas de jet illégal de déchets dans les eaux territoriales des États-Unis, de Puerto 
Rico, des îles Vierges américaines ont été rapportés, tandis que très peu d'amendes ou de condamnations effectives ont été infligées aux paquebots responsables, en raison de la protection que confère le régime de pavillon de complaisance; récemment, cependant, certaines compagnies ont commencé à poser des actes concrets de respect des réglementations environnementales, nationales et internationales, dans les eaux internationales et nationales (Dowling, 2006 : 14-15), afin de préserver l'environnement marin qui est l'une des principales ressources productives de la croisière.

\section{Conclusion}

Bien que les autorités touristiques caribéennes, réunies au sein de la Caribbean Tourism Organization, envisagent le tourisme de croisière comme un axe stratégique de développement durable potentiel de leur région, l'étude proposée ici incite à la prudence. D'abord et principalement parce que la région ne joue qu'un rôle marginal dans la stratégie d'offre de l'industrie: ce sont les compagnies de croisière qui seules conçoivent les stratégies d'innovation, de différenciation, de marketing des produits qui orientent la demande ainsi que le marché ; ensuite, parce que les compagnies de croisière sont détenues quasi exclusivement par des capitaux extra-caribéens. Enfin, parce que les paquebots battant pavillon de complaisance panaméen et libérien, pour la plupart, puis accessoirement bahaméen ou bermudien, échappent aux législations nationales caribéennes en matière d'environnement, de travail, de fiscalité, etc.

Il en résulte pour les économies caribéennes un impact ténu:

- sur le plan économique, bien que les compagnies réalisent d'importants profits, ceux-ci rémunèrent quasi exclusivement des capitaux extra-caribéens; et quoique les dépenses globales des croisiéristes soient notables, la part de ces dépenses qui passe directement dans les économies nationales reste faible tout autant que les effets indirects et induits liés en termes de revenus;

- sur le plan social, les retombées régionales de l'activité de croisière en termes d'emplois sont insignifiantes au regard des revenus que génère la croisière : d'abord, le personnel caribéen employé à bord des paquebots reste très minoritaire et est de surcroît mal rétribué avec des conditions de travail pénibles; ensuite, les emplois ter- restres directs et indirects dus à l'activité de croisière apparaissent largement faibles, au moins au regard de ceux générés par le tourisme de séjour ;

- sur le plan environnemental, d'importantes externalités négatives sont causées par l'industrie de croisière, lesquelles risquent de se retrouver à la charge des territoires.

Enfin, et toujours au rang des externalités négatives, il faut noter que les orientations stratégiques en cours dans l'industrie, faisant des paquebots des destinations touristiques en soi et engendrant progressivement dans la Caraibe de nouveaux "territoires touristiques sans terre » capables de capter les aménités géographiques clés à la base de la demande touristique de la région (mer, soleil), transforment graduellement l'industrie de croisière en concurrente sérieuse des destinations touristiques terrestres traditionnelles. En cela, le développement de l'industrie de croisière dans la Carailbe constitue, à terme, une menace pour l'activité touristique traditionnelle de la région, alors que cette activité est aujourd'hui le pilier majeur du développement de la région : des doutes sérieux peuvent être émis quant à la viabilité d'une stratégie de développement touristique durable de la Caraïbe fondée sur l'expansion de l'activité de croisière.

Kinvi Logossah est maitre de conférences et directeur de recherche au Centre d'études et de recherche en économie, gestion, modélisation et informatique appliquée, Université des Antilles et de la Guyane (Martinique).

\section{Notes}

1 Au sens de ces auteurs, un petit pays est défini par une démographie inférieure à un million d'habitants et un pays à spécialisation touristique par un ratio recettes touristiques sur PIB supérieur à $10 \%$.

2 Un gallon vaut 4,546 litres au Canada et en Grande-Bretagne et 3,785 litres aux États-Unis.

\section{Bibliographie}

Aghion, Philippe, et Peter Howitt (1998), Endo_genous Growth Theory, Cambridge, Massachusetts, Massachusetts Institute of Technology / MIT.

Brau, Rinaldo, Alessandro Lanza, et Francesco Pigliaru (2003), How Fast Are Tourism Countries Growing: The Cross Country Evidence, Social Science Research Network Electronic Paper Collection, [http://papers.ssrn.com/abstract_id=XXXXXX].

Braun, Bradley, James Xander, et Kenneth White (2002), « The Impact of the Cruise Industry on a Region's Economy: A Case Study of Port Canaveral, Florida ", Tourism Economics, vol. 8, n`3, p. 281-288.
CMT (2003a), Bilan 2003 du tourisme à la Martinique, Livre I, Schoelcher, Martinique.

CMT (2003b), Bilan 2003 du tourisme à la Martinique, Livre II, Schoelcher, Martinique.

CTO (2006a), Key Indicators of Caribbean Tourism, [oCTO2004.pfd], [www.onecaribbean.org].

CTO (2006b), Key Indicators of Caribbean Tourism, [CTOreports2004.pfd], [www.onecaribbean.org].

DRCE (Direction régionale du commerce extérieur, 2004), Statistiques du commerce extérieur, Martinique.

Dowling, Ross K. (2006), "The Cruise Industry », dans Ross K. Dowling (dir.), Cruise Ship Tourism, Cab International, Cambridge, Massachusetts.

Grossman, Gene, et Elhanan Helpman (1991), Innovation and Growth in the World Economy, MIT Press, Cambridge USA.

Lester, Jo-Anne, et Clarke Weeden (2004), "Stakeholders, the Natural Environment and the Future of the Caribbean Cruise Tourism ", International Journal of Tourism Research, $n^{\circ}$ 6, p. 39-50.

Logossah, Kinvi, et Marie-Antoinette Maupertuis (2007), "La spécialisation touristique des petites économies insulaires en développement est-elle une voie de croissance durable? ", Revue d'économie régionale et urbaine, $\mathrm{n}^{\circ} 1$, p. 35-57.

Marques, Bruno (2005), «Tourisme durable et croissance: L'expérience des pays de la Caraïbe ", dans Kinvi Logossah et Jean-Michel Salmon (dir.), Tourisme et développement durable, Publibook, Paris, p. 209-218.

Ocean Conservancy (2002), Cruise Control, [http://montereybay.noaa.gov/sac/2002/0607 02/occr53102.pdf].

Pricewaterhousecoopers et Brea (2001), Cruise Industry's Economic Impact on the Caribbean, [http://www.f-cca.com/downloads/carib_im pact.pdf].

Seidl, Andy, Fiorella Guilliano, et Lawrence Pratt (2006), "Cruise Tourism and Community Economic Development in Central America and Caribbean: The Case of Costa Rica", PASOS, Revista de Tourismo y Patrimonio Cultural, vol. 4, n², p. 213-224.

Thomas, Stephen Jr. (2004), « State Regulation of Cruise Ship Pollution: Alaska's Commercial Passenger Vessel Compliance Program As a Model for Florida ", Journal of Transnational Law and Policy, vol. 13, n², p. 533-557.

Wood, Robert E. (2004), « Global Currents: Cruise Ships in the Caribbean Sea", dans David Duval (dir.), Tourism in the Caribbean: Trends, Developments, Prospects, Routledge, New York, p. 152-171.

Wood, Robert E. (2005), Neoliberal Globalization: The Cruise Ship Industry As a Paradigmatic Case, draft, Rutgers University, Camden campus.

WTO (2004), Tourism Market Trends, [WTO.org]. WTO (2005), Baromètre OMT du tourisme mondial, vol. 3, n², juin.

WTO (2006), Baromètre OMT du tourisme mondial, octobre. 